\section{Infection with cytomegalovirus is not associated with premature mortality}

\author{
Stuart P. Adler, ${ }^{1}$ Al M. Best, ${ }^{2}$ \\ Beth Marshall, ${ }^{1}$ George W. Vetrovec ${ }^{3}$ \\ 'Division of Infectious Diseases, \\ Department of Pediatrics; ' Department \\ of Biostatistics; ${ }^{3}$ Division of Cardiology, \\ Department of Internal Medicine, Medical \\ College of Virginia Campus of Virginia \\ Commonwealth University, Richmond \\ VA, USA
}

\section{Abstract}

Over $90 \%$ of the world's population acquires a cytomegalovirus (CMV) infection. This infection, although asymptomatic or self-limiting, is a major burden to the immune system. For this reason, and because CMV immunization is possible, determining whether CMV can cause reduced longevity, particularly among those with coronary artery disease, is important and previous reports have been conflicting. Thus our objective was to assess the association between $\mathrm{CMV}$ infection as defined serologically and antibody levels against CMV and longterm survival (18 years). We completed a prospective observational cohort study of 915 consecutive patients (mean age 58 years) undergoing coronary angiography. CMV immunoglobulin levels were measured at baseline using either a whole cell CMV antigen or a purified protein antigen (gB). After adjustment for potentially confounding variables (age, race, gender, body mass index, the presence or absence of coronary artery disease, the number of diseased vessels, diabetes, renal disease, hypertension, dialysis, congestive heart failure, and the maximum percent reduction in luminal diameter), Cox's proportional hazards models showed no association between CMV seropositivity or levels of antibodies against CMV by either assay and longevity for both patients with or without coronary artery disease (CAD) nor for those under or over 70 years of age at baseline. Our observations suggest that universal immunization against CMV may not improve longevity.

\section{Introduction}

Cytomegalovirus (CMV) is a ubiquitous viral infection that eventually infects over $90 \%$ of the world's human population, although the rate at which CMV is acquired varies widely depending on location and a variety of social and demographic factors. For the vast majority of infected individuals, CMV causes no obvious disease but it is a serious pathogen for pregnant women and their fetuses, and for those with depressed cellular immunity. Among infected asymptomatic healthy individuals, CMV infections do place a significant burden upon the immune system and this burden increases with age. Both CMV antibody titers and percentage of $\mathrm{CD}^{+}$cells targeting $\mathrm{CMV}$ increase with age, suggesting a chronic persistent subclinical infection. ${ }^{1,2}$

Recent studies have observed an association between CMV infections and high titer antibodies, and premature mortality in elderly immunocompetent adults. ${ }^{3-5}$ The reason for this is unclear but CMV does play a role in the development of certain types of coronary atherosclerosis. Following cardiac transplantation, those patients with CMV infections are at significantly greater risk of developing accelerated post transplantation coronary atherosclerosis than are patients not infected with CMV. ${ }^{6}$ Epidemiological data suggest that a prior CMV infection is a risk factor for restenosis of coronary arteries following coronary atherectomy. ${ }^{?}$ Although the exact mechanism by which CMV promotes atherosclerotic coronary occlusion is unknown, some hypotheses have been made. ${ }^{8}$

Less clear, however, is the role, if any, that CMV plays in the development of atherosclerotic heart disease. Several studies have identified high antibody titers to CMV as a risk factor for the development of coronary atherosclerosis. ${ }^{9,10}$ Other studies have not confirmed an association between CMV infections and/or high antibody titers and coronary artery disease. ${ }^{11-13}$

Although there is currently no licensed CMV vaccine, CMV is a vaccine preventable infection. ${ }^{14}$ Current vaccine strategies contemplate immunizing women of child-bearing age and transplant recipients. If, however, CMV is associated with accelerated coronary artery disease and/or decreased longevity, then universal immunization against CMV may be desirable. Therefore, to determine whether CMV seropositivity and/or the titer of antibodies to CMV antigens AD169 and glycoprotein B (the major CMV envelope glycoprotein containing neutralizing epitopes against CMV) predicts long-term survival, we studied the survival of over 900 successive adult patients with and without angiographically proven coronary artery disease.

\section{Materials and Methods}

\section{Subjects and study design}

This study involved 920 consecutive adult patients undergoing coronary angiography at the Medical College of Virginia Hospital
Correspondence: Stuart P. Adler, Department of Pediatrics, Medical College of Virginia Campus of Virginia Commonwealth University, P 0 Box 980163, Richmond, VA 23298, USA.

E-mail: sadler@vcu.edu

Key words: cytomegalovirus, coronary artery disease, mortality.

Conflict of interest: the authors report no conflicts of interest.

Received for publication: 18 August 2011. Accepted for publication: 30 October 2011.

This work is licensed under a Creative Commons Attribution NonCommercial 3.0 License (CC BYNC 3.0).

(C) Copyright S.P. Adler et al., 2011

Licensee PAGEPress, Italy

Infectious Disease Reports 2011; 3:e17

doi:10.4081/idr.2011.e17

between November 1991 and June 1992 Details on the study methods and sampling design have been previously published. ${ }^{11}$ The study was a prospective observational cohort study in which we examined the serum CMV immunoglobulin (IgG) antibody and the risk of mortality. Blood was drawn from each patient by venipuncture, collected into a $10 \mathrm{~mL}$ nonheparinized vacuum tube and left to clot at room temperature. The serum samples were centrifuged and sera removed, aliquoted, and stored frozen at $-70^{\circ}$ until being assayed. Five subjects without baseline serum specimens were excluded leaving 915 patients for analysis. For each patient, a case report form was also collected which listed a variety of demographic and health data, and catheterization results. The study was approved by the VCU Committee for the Conduct of Human Research.

Patient information obtained included gender, race, height, weight, birth date and cholesterol. Body mass index (BMI) and age at angiography were obtained from this information. Patients were considered to have coronary artery disease (CAD) if there was a reduction in luminal diameter of any coronary artery of more than $50 \%$ or if there had been a previous coronary artery bypass graft or previous percutaneous transluminal angioplasty. The number of diseased vessels ( $>50 \%$ blockage) was counted and the severity of disease was assessed as the maximum blockage of the four coronary arteries. Additional risk factors were also recorded: diabetes, impaired renal function, hypertension, dialysis, or congestive heart failure.

In 2001 and 2010, patient deaths were confirmed using the social security death index, patient's name, social security number, and last known residence. Those still alive as of 
May 2010 were included in the survival analyses as a censored observation. The survival time was determined as the number of years surviving after catheterization for the primary analysis. This was calculated as the number of days between the catheterization date and the date of death (or May $1^{\text {st }} 2010$ for censored observations) divided by 365.25 . Thus, this is an over 18-year mortality determination. For 920 study participants, the date of death was known for $61 \%$; 565 died. For the secondary end-point, survival time was determined by the number of years surviving since birth.

\section{Serological assays}

Serum IgG antibodies to CMV were first measured quantitatively with an enzyme immunoassay (EIA) using AD169 as previously described. ${ }^{11,15,16}$ Calibrator sera and positive and negative control sera were included on each plate. All sera were diluted 1:20. Absorbance was measured on a dual wavelength Vmax Microelisa Auto Reader (Molecular Devices, Palo Alto, CA, USA). Absorbance values (test absorbance minus control absorbance) of the calibrator sera were plotted against a standard number of antibody units determined by end-point dilution, and the number of antibody units for the test serum was calculated from the regression equation. Greater than 50 antibody units was considered positive. The correlation coefficient of the calibrator sera was over 0.95 in all tests. IgG antibodies to purified CMV glycoprotein B were assayed as previously described. ${ }^{17}$ Briefly, to measure IgG antibodies to $\mathrm{gB}$ in sera, sera were diluted to $1: 3200$ and 1:6400 in duplicate in PBST with 3\% BSA, and $100 \mu \mathrm{L}$ of each dilution were added per microwell precoated with $10 \mu \mathrm{g} / \mathrm{mL}$ of purified gB (Chiron Corp., Emeryville, CA, USA) in carbonate buffer (ph 9.6) or $100 \mu \mathrm{L}$ of control antigen $(10 \mu \mathrm{g} / \mathrm{mL}$ of bovine serum albumin (BSA). After $90 \mathrm{~min}$ at $37^{\circ} \mathrm{C}$, the microwells were washed four times with PBST and then $100 \mu \mathrm{L}$ of a 1:500 dilution of alkaline phosphatase (AP) conjugated goat antihuman immunoglobulin G (Tago, Inc., Burlingame, CA, USA) in PBST with $3 \%$ BSA was added to each microwell. After $90 \mathrm{~min}$ at $37^{\circ} \mathrm{C}$, the microwells were washed four times with PBST and then $100 \mu \mathrm{L}$ of $p$-nitrophenyl phosphate (Sigma Chemical Co., St. Louis, M0, USA), $1 \mathrm{mg} / \mathrm{mL}$, in $10 \%$ diethanolamine (pH 9.6) was added to each well. The plates were incubated at room temperature until an optical density of 0.200 at $405 \mathrm{~nm}$ had developed with a 1:6,400 dilution of a CMV positive serum (30-60 min.). The difference in absorption at $405 \mathrm{~nm} / 490 \mathrm{~nm}$ between the control wells (coated with BSA) and the wells coated with $\mathrm{gB}$ was measured on a dual wavelength Vmax Microelisa Auto Reader (Molecular Devices, Palo Alto, CA, USA). All assays were run in duplicate. A standard curve for the association between optical density units and serial dilutions of a pooled intravenous immunoglobulin (IVIG) preparation (Sandoz, East Hanover, NJ, USA) was established and previously published..$^{11}$ For each subject, the number of IVIG antibody units per 1:3200 dilution of serum was calculated with an antibody unit defined as the natural log of the reciprocal of the dilution of IVIG required to produce the observed optical density units.

\section{Statistical analysis}

Study participants' baseline characteristics were compared across categories of CMV antibody concentration using either ANOVA for continuous variables or the $\chi^{2}$ test for binary variables. The primary analysis compared survival time between five antibody concentration groups using a Kaplan-Meier survival analysis. Additional analyses tested for a difference in survival time between those antibody negative and those positive, and also tested for a trend in survival time using CMV antibody as a continuous predictor in a Cox's proportional hazards model. An additional primary analysis compared survival time between the five antibody concentration groups after adjusting for all other risk factors in a Cox's proportional hazards model. These risk factors were determined by first screening the demographic and medical characteristics using a Cox's proportional hazards model for the continuous risk factors or a Kaplan-Meier survival analysis for the nominal risk factors. Those risk factors significantly associated with survival time were included in the adjusted Cox's proportional hazards analysis.

Additionally, since previous studies indicated CMV infection as a risk factor for earlier mortality in older adults, similar Cox's proportional hazards models were used to test this association only in individuals who were at least 70 years old at the beginning of the study.

All $P$ values were derived from two-tailed tests. Analyses were conducted using SAS software (JMP version 9.0, SAS Institute Inc., Cary, NC, USA).

\section{Results}

The demographic and medical characteristics of the patients included in the prospective cohort are shown in Table 1. The mean age of patients was 58.4 years $(\mathrm{SD}=12.2)$ and the mean BMI was $27.8 \mathrm{lbs} / \mathrm{in}^{2}(\mathrm{SD}=5.9)$. The patients were $59 \%$ Caucasian and $56 \%$ male. Based on angiography, $60 \%$ of the patients had newly diagnosed coronary artery disease (CAD) and another $10 \%$ had had previous coronary artery disease based on a previous coronary artery bypass graft or previous percuta- neous transluminal angioplasty. So overall, at baseline, $70 \%$ of the patients had CAD. Forty percent of patients had less than two coronary vessels obstructed (> 50\%) and 60\% had one or more of the following risk factors: diabetes (26\%), impaired renal function (4\%), hypertension (51\%), dialysis (2\%), or congestive heart failure (5\%). The average maximal reduction in arterial luminal diameter was $60 \%$.

The CMV IgG concentration to AD169 was grouped into five groups: a seronegative group and four additional groups according to quartiles of antibody concentration in the seropositive range. Since CMV antibody concentration may be associated with the other characteristics included in the table, we tested for a significant difference between the five quintiles. There was a significant difference in average age across the five quintiles (ANOVA $\mathrm{P}=0.02$ ), and this seems to be accounted for by the younger seronegative subjects. Similarly, lower antibody concentration is associated with being white and being male $\left(\chi^{2}\right.$ Ps $\left.<0.01\right)$. There were fewer patients in the seronegative group who had any risk factor $(\mathrm{P}=0.2)$. There was no apparent difference between the five groups of antibody concentrations according to BMI, cholesterol, the presence or absence of $\mathrm{CAD}$, or the number of diseased vessels.

For subjects between 40 and 70 years of age at baseline, similar results were obtained for the antibody assay using $\mathrm{gB}$ antigen (Table 2).

Since there are two outcomes (survival years after angiography, and total survival years after birth) and two CMV antibody measures (IgG concentration using AD169 and IgG concentration using the $\mathrm{gB}$ antigen), there are four main analyses to consider. Additionally, similar analyses will be reported in a subset of older adults.

\section{Survival years after angiography versus CMV IgG to AD169}

To test whether CMV IgG concentration to $\mathrm{AD} 169$ is associated with a higher risk of mortality we first used the survival time from the day of coronary angiography. A Kaplan-Meier survival analysis comparing the five quintiles of antibody concentration showed no significant difference (log-rank $\chi^{2}=2.2, \mathrm{P}=0.69$ ). The median survival time across all groups was 12.9 years and the largest difference between groups was between the seronegative subjects (median $=14.4$ years) and the first quartile (median $=12.0$ years)

To assess the independent effect of CMV infection on mortality, it is necessary to adjust for potential confounders. This was performed using Cox's proportional hazards model. To identify the potential confounders, we identified significant predictors of mortality by screening each of the characteristics listed in Table 1. Continuous characteristics (e.g. age) 
were screened using a Cox's proportional hazards analysis and the nominal characteristics (e.g. male) were screened using a KaplanMeier survival analysis. These screening results are shown in Table 3. Older subjects are subject to a higher mortality risk $(\mathrm{P}<0.01)$ as were those with higher BMI $(\mathrm{P}<0.01)$. Those with $\mathrm{CAD}$ have a median survival time of 12.3 years whereas those without CAD survive a median of 15.9 years. There is a similar negative risk for those newly diagnosed with CAD at baseline. An increasing number of diseased vessels, the presence of a risk factor, or a greater reduction in luminal artery diameter carries an increased mortality risk. The screening did not indicate that race, gender, or cholesterol were significant risk factors, so they were not included in the multivariate Cox's proportional hazards model.

The adjusted test for the effect of CMV infec- tion on post-angiography survival time included the following potential confounders: age, BMI, CAD, number of diseased vessels, the presence of any risk factor, and the maximum percent of reduction in luminal diameter. Additionally, the five quartiles of CMV antibody concentration were included in the model to test the primary aim of the study. Since the sample size was smaller for those newly diagnosed with $\mathrm{CAD}$, all subjects with $\mathrm{CAD}$ were included in the Cox's model. As shown in Table 3 , all of the confounders except the percent reduction in luminal diameter remained significant in the multivariate model. However, even after adjustment for these confounders, there was no evidence that the five quintiles of CMV antibody concentration described significantly different mortality groups $(\mathrm{P}>0.9)$. The same result was found when excluding those with previously diagnosed $\mathrm{CAD}$ patients

\section{( $\mathrm{P}>0.8$, data not shown $)$.}

The other three tests in Table 3 represent the results of using other ways of considering CMV IgG antibody concentration. If instead of 5 quintiles, the concentration is considered a continuous predictor, no significant risk is indicated $(\mathrm{P}>0.5)$. If antibody concentration is considered as seronegative or seropositive, there is no significant difference $(\mathrm{P}>0.5)$, or if the highest quintile is considered the binary risk group, there is no significant difference $(\mathrm{P}=0.6)$.

\section{Survival years after birth versus CMV IgG to AD169}

The adjusted test for the effect of CMV infection on survival years included the same confounders. The median survival age was 75.8 years. The results for these analyses are shown in Table 3 . The same pattern of relationships

Table 1. Baseline characteristics of the study patients by cytomegalovirus IgG concentration quintiles using AD169 antigen.

\begin{tabular}{|c|c|c|c|c|c|c|c|c|}
\hline Characteristic & \multicolumn{8}{|c|}{$\begin{array}{l}\text { CMV IgG concentration to AD169* } \\
\text { five quintiles }\end{array}$} \\
\hline Mean age, years ${ }^{\circ}$ & 915 & 58.4 & 55.8 & 59.9 & 58.4 & 59.1 & 59.1 & 0.02 \\
\hline Mean BMI, lbs/in ${ }^{2}$ & 845 & 27.8 & 28.0 & 27.8 & 27.0 & 27.4 & 28.8 & 0.06 \\
\hline White race, $\% *$ & 847 & 59.4 & 77.7 & 47.0 & 53.6 & 60.7 & 57.1 & $<.01$ \\
\hline Male, $\%$ & 913 & 56.4 & 71.0 & 56.9 & 57.5 & 51.1 & 45.3 & $<.01$ \\
\hline Cholesterol, mg \% & 758 & 208.2 & 210.7 & 217.6 & 215.9 & 219.3 & 221.5 & 0.53 \\
\hline $\mathrm{CAD}, \%$ & 915 & 70.1 & 69.9 & 65.4 & 72.2 & 71.6 & 71.8 & 0.60 \\
\hline Newly diagnosed $\mathrm{CAD}^{\#} \%$ & 685 & 60.1 & 57.7 & 55.8 & 63.0 & 62.0 & 62.5 & 0.65 \\
\hline Number of diseased vessels & 915 & 1.2 & 1.3 & 1.3 & 1.5 & 1.3 & 1.4 & 0.77 \\
\hline Any risk factor $\$$ & 915 & 60.0 & 49.7 & 61.2 & 60.6 & 67.2 & 61.3 & 0.02 \\
\hline \% luminal reduction & 915 & 59.5 & 60.6 & 55.6 & 59.4 & 61.3 & 60.7 & 0.72 \\
\hline
\end{tabular}

CMV, cytomegalovirus; BMI, body mass index; CAD, coronary artery disease. *Values $>50$ units were considered seropositive. The classification of subjects into antibody concentration quintiles were: those who were CMV seronegative and those who were in the first, second, third, and fourth quartiles, respectively. The five antibody groups compared using either ANOVA or $\chi^{2}$. ${ }^{\circ}$ Significant difference between the 5 antibody groups in the specified characteristic, $\mathrm{P}<0.05$. Excluding patients with previous coronary artery bypass graft or previous percutaneous transluminal angioplasty. ${ }^{\circledR}$ Risk factors: diabetes, impaired renal function, dialysis, hypertension, or congestive heart failure.

Table 2. Baseline characteristics of the study patients between 40 and 70 years of age using $\mathrm{gB}$ antigen.

\begin{tabular}{|c|c|c|c|c|c|c|c|c|}
\hline \multirow[b]{2}{*}{ Characteristic } & \multicolumn{8}{|c|}{$\begin{array}{l}\text { CMV IgG concentration to } \mathrm{gB}^{*} \\
\text { five quintiles }\end{array}$} \\
\hline & $\begin{array}{l}\text { All pi } \\
\text { n. }\end{array}$ & $\begin{array}{l}\text { ipants } \\
\text { Mean }\end{array}$ & $\begin{array}{r}\text { Neg. }<5 \\
(\mathrm{n}=118)\end{array}$ & $\begin{array}{r}5-8.1 \\
(n=135)\end{array}$ & $\begin{array}{l}8.2-8.61 \\
(\mathrm{n}=135)\end{array}$ & $\begin{array}{r}8.62-9.15 \\
(\mathrm{n}=135)\end{array}$ & $\begin{array}{r}>9.15 \\
(\mathrm{n}=138)\end{array}$ & P* \\
\hline Mean age, years ${ }^{\circ}$ & 661 & 55.6 & 52.9 & 55.4 & 55.6 & 57.1 & 56.6 & $<.01$ \\
\hline Mean BMI, lbs/in² & 613 & 28.5 & 28.6 & 28.3 & 28.8 & 28.9 & 28.1 & 0.83 \\
\hline White race, $\% *$ & 622 & 57.2 & 82.3 & 46.1 & 48.0 & 53.5 & 59.1 & $<.01$ \\
\hline Male, $\%$ & 659 & 57.4 & 76.3 & 43.7 & 45.9 & 57.5 & 65.7 & $<.01$ \\
\hline Cholesterol, mg \% & 557 & 210.3 & 201.1 & 210.1 & 214.8 & 209.3 & 215.0 & 0.37 \\
\hline $\mathrm{CAD}, \%$ & 661 & 69.4 & 67.8 & 67.4 & 65.2 & 74.8 & 71.7 & 0.44 \\
\hline Newly diagnosed CAD & 495 & 59.2 & 54.2 & 56.4 & 58.4 & 65.7 & 60.6 & 0.56 \\
\hline Number of diseased vessels & 661 & 1.1 & 1.1 & 1.0 & 1.1 & 1.2 & 1.3 & 0.33 \\
\hline Any risk factor ${ }^{\S}, \%^{\circ}$ & 661 & 61.6 & 49.2 & 68.9 & 65.9 & 65.2 & 57.2 & 0.01 \\
\hline \% luminal reduction & 661 & 58.5 & 58.5 & 55.0 & 56.1 & 62.3 & 60.7 & 0.61 \\
\hline
\end{tabular}

CMV, cytomegalovirus; BMI, body mass index; CAD, coronary artery disease. *Values $>5$ were considered seropositive. The classification of subjects into antibody concentration quintiles were: those who were CMV seronegative and those who were in the first, second, third, and fourth quartiles, respectively. The five antibody groups compared using either ANOVA or $\chi^{2}$. ${ }^{\circ}$ Significant difference between the 5 antibody groups in the specified characteristic, $\mathrm{P}<0.05$. "Excluding patients with previous coronary artery bypass graft or previous percutaneous transluminal angioplasty. ${ }^{\circledR}$ Risk factors: diabetes, impaired renal function, dialysis, hypertension, or congestive heart failure. 
with the confounders was found. And the analyses also indicated no support for a relationship with CMV antibody levels (Ps> 0.7).

\section{Survival years versus $\mathrm{CMV}$ IgG to gB}

We next examined survival in relation to the levels of IgG to CMV glycoprotein B in patients who were aged 40 to 70 years of age at baseline. The adjusted tests for the effect of antibody infection on survival years are shown in Table 3. The analyses of survival after birth or angiography indicate no support for a relationship with CMV infection (Ps>0.15).

\section{Survival years versus CMV IgG antibody to AD169 for the elderly}

We next examined survival in all individuals who were at least 70 years of age $(n=178$, Table 4). Cox's proportional hazards analysis was used to test for relationships with CMV IgG to AD169. The same pattern of relationships with the confounders was found and the analysis also indicated no support for a relationship with CMV for either measure of survival time (Ps $>0.14$ for years after angiography and $\mathrm{Ps}>0.16$ for total survival time).

In summary, in none of the analyses using different survival time measures and different CMV antibody measures did we find any evi- dence that CMV infection increased the risk of mortality. This was true in the larger study of all 913 patients and in the 178 elderly patients.

\section{Discussion}

Our data from a large cohort of non-transplant patients undergoing cardiac catheterization suggest that CMV infection is unlikely to be an important independent risk factor for premature death both among patients with or without atherosclerotic heart disease. Our study has several strengths. First, our study included a large sample size of 915 consecutive patients undergoing coronary angiography and used an objective quantitative measure, angiography results, to define coronary artery disease. Second, our sample included two racial groups, both genders, and diverse ages and patients with and without coronary artery disease to asses the association between CMV infection, anti-CMV antibody levels, and longevity. Third, we used two antigens (AD169 whole cell antigen and a purified protein antigen, gB) to measure the levels of IgG to CMV at baseline. The $\mathrm{gB}$ antigen used was identical to the protein used in an effective vaccine and antibodies to this antigen correlate with neu- tralizing activity whereas the assay using whole cell AD169 antigen does not. ${ }^{14}$ Fourth, our follow-up time for assessing survival was at least 18 years; far longer than previously published studies. For these reasons, it is likely our observations reflect what would have been observed in a true population-based study with prolonged follow up.

A possible limitation of our study was that, as previously reported by others, we did not include large subgroups of patients for whom there may have be an association between survival and CMV infection associated with high titer antibodies. ${ }^{3,5,8}$ For example, we did not measure non-specific markers of inflammation such as C-reactive protein or interleukin6 , smoking, or chronic concurrent infections such as Chlamydia, hepatitis, herpes, etc. These factors, if present in any given patient, may interact with CMV and be associated with reduced longevity, as has been previously suggested. ${ }^{5,8}$ Nor did we include exclusively elderly patients or only those with coronary artery disease, as in other studies., ${ }^{3,4,5,13,18}$

Our observations appear to support previous studies and contradict others. Three recent studies did observe an association between those with high antibody titers to CMV and reduced longevity. ${ }^{3-5}$ These studies enrolled only elderly subjects. In one, the mean age was 80

Table 3. Survival analysis of patients by two measures of cytomegalovirus antibody.

\begin{tabular}{|c|c|c|c|c|c|c|c|c|c|}
\hline \multirow[b]{3}{*}{ Characteristic } & \multirow{2}{*}{\multicolumn{3}{|c|}{ Screening analyses* }} & \multicolumn{2}{|c|}{$\begin{array}{l}\text { Survival years } \\
\text { after angiography }\end{array}$} & \multirow{2}{*}{\multicolumn{2}{|c|}{ Adjusted analyses $^{\circ}$}} & & \\
\hline & & & & & & & & & \multirow[b]{2}{*}{$\mathbf{P}$} \\
\hline & $\mathbf{N}$ & $\chi^{2}$ & P & risk ratio & $\chi^{2}$ & P & risk ratio & $\chi^{2}$ & \\
\hline Age, years & 913 & 124.1 & $<.01$ & 1.036 & 77.7 & $<.01$ & 0.913 & 248.8 & $<.01$ \\
\hline BMI, lbs/in ${ }^{2}$ & 845 & 11.6 & $<.01$ & 0.981 & 5.2 & 0.02 & 0.981 & 5.4 & 0.02 \\
\hline White race & 851 & 0.6 & 0.44 & & & & & & \\
\hline Male & 915 & 0.6 & 0.42 & & & & & & \\
\hline Cholesterol, mg\% & 762 & 0.5 & 0.47 & & & & & & \\
\hline $\mathrm{CAD}$ & 915 & 8.1 & $<.01$ & 0.580 & 6.0 & 0.01 & 0.545 & 7.2 & 0.01 \\
\hline Newly diagnosed CAD & 687 & 9.5 & $<.01$ & & & & & & \\
\hline Number of diseased vessels & 915 & 31.6 & $<.01$ & 1.167 & 7.2 & 0.01 & 1.162 & 6.5 & 0.01 \\
\hline Any risk factor & 899 & 7.6 & 0.01 & 1.313 & 8.4 & $<.01$ & 1.290 & 7.2 & 0.01 \\
\hline$\%$ luminal reduction & 915 & 19.6 & $<.01$ & 1.004 & 1.7 & 0.19 & 1.005 & 3.5 & 0.06 \\
\hline \multicolumn{10}{|l|}{ CMV IgG to AD169 } \\
\hline Five quintiles & 913 & 2.2 & 0.69 & 0.905 & 0.9 & 0.93 & 0.956 & 0.9 & 0.92 \\
\hline Concentration & 913 & 0.8 & 0.38 & 1.000 & 0.4 & 0.53 & 0.922 & 0.1 & 0.74 \\
\hline Seropositive & 913 & 0.2 & 0.17 & 0.935 & 0.4 & 0.55 & 0.962 & 0.1 & 0.74 \\
\hline Highest Quintile & 913 & $<.1$ & 0.86 & 0.944 & 0.3 & 0.60 & 0.982 & $<.1$ & 0.87 \\
\hline \multicolumn{10}{|l|}{$C M V \operatorname{Ig} G$ to $g B^{\S}$} \\
\hline Five quintiles & 658 & 4.5 & 0.34 & 0.792 & 5.6 & 0.23 & 0.845 & 6.8 & 0.15 \\
\hline Concentration & 658 & $<.1$ & 0.93 & 0.978 & 1.9 & 0.17 & 0.982 & 1.3 & 0.26 \\
\hline Seropositive & 658 & 0.1 & 0.71 & 0.875 & 0.9 & 0.36 & 0.904 & 0.5 & 0.49 \\
\hline Highest quintile ${ }^{\sharp}$ & 658 & 1.0 & 0.32 & 0.857 & 1.4 & 0.24 & 0.903 & 0.6 & 0.43 \\
\hline
\end{tabular}

CMV, cytomegalovirus; BMI, body mass index; $\mathrm{CAD}$, coronary artery disease.

*The primary outcome was the number of years surviving after angiography (see Materials and Methods). Screening was performed using either Kaplan-Meier survival analysis or Cox's proportional hazards.

${ }^{\circ}$ The secondary outcome was the number of years surviving after birth. Adjusted analyses used the significant risk factors (age, BMI, CAD, number of diseased vessels, percent luminal reduction and one of the CMV antibody measures listed in the bottom of the table.

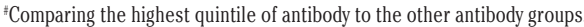

${ }^{8}$ Includes only patients between 40 and 70 years of age. 
Table 4. Survival analysis of patients 70 years or older by cytomegalovirus igG to AD169.

\begin{tabular}{|c|c|c|c|c|c|c|}
\hline \multirow[b]{2}{*}{ Characteristic } & \multicolumn{3}{|c|}{$\begin{array}{l}\text { Years after } \\
\text { angiography* } \\
(\mathrm{n}=178)\end{array}$} & \multicolumn{3}{|c|}{$\begin{array}{l}\text { Years after birth } \\
(\mathrm{n}=171)\end{array}$} \\
\hline & Risk ratio & $\chi^{2}$ & $\begin{array}{l}\text { juste } \\
\text { P }\end{array}$ & $\begin{array}{l}\text { Inalyses } \\
\text { Risk ratio }\end{array}$ & $\chi^{2}$ & $\mathbf{P}$ \\
\hline Age, years & 1.030 & 2.3 & 0.13 & 0.913 & 22.8 & $<.01$ \\
\hline BMI, lbs/in ${ }^{2}$ & 0.946 & 7.8 & 0.01 & 0.981 & 8.5 & $<.01$ \\
\hline $\mathrm{CAD}$ & 0.766 & 0.2 & 0.62 & 0.545 & 0.6 & 0.44 \\
\hline Number of diseased vessels & 1.327 & 7.5 & 0.01 & 1.162 & 4.4 & 0.04 \\
\hline Any risk factor & 1.004 & 0.0 & 0.98 & 1.290 & 0.2 & 0.69 \\
\hline \% luminal reduction & 0.995 & 0.6 & 0.45 & 1.005 & 0.0 & 0.89 \\
\hline CMV IgG to AD169 & & & & & & \\
\hline Five quintiles & 0.905 & 5.2 & 0.27 & 0.756 & 3.9 & 0.42 \\
\hline Concentration & 0.999 & 2.2 & 0.14 & 0.999 & 2.0 & 0.16 \\
\hline Seropositive & 0.711 & 0.5 & 0.47 & 0.856 & 0.5 & 0.50 \\
\hline Highest quintile ${ }^{\#}$ & 0.797 & 1.2 & 0.27 & 0.815 & 0.9 & 0.33 \\
\hline
\end{tabular}

CMV, cytomegalovirus; BMI, body mass index; CAD, coronary artery disease.

*The primary outcome was the number of years surviving after angiography (see Materials and Methods).

${ }^{\circ}$ The secondary outcome was the number of years surviving after birth. Adjusted analyses used the significant risk factors (age, BMI, CAD, number of diseased vessels, percent luminal reduction and one of the CMV antibody measures listed in the bottom of the table.

"Comparing the highest quintile of antibody to the other antibody groups.

years. A second enrolled only Latino subjects aged 60 years or older, and the third enrolled only women between 70 and 79 years of age. In another study of 890 patients with a mean age of 65 years and significant coronary disease established by angiography, an association between CMV seropositivity and death or myocardial infarction was observed over three years. ${ }^{8}$ In this study, however, there was a strong independent association with death or myocardial infarction and chronic infection with H. pylori, Chlamydia pneumoniae, herpes simplex, and evidence of chronic inflammation as measured by C-reactive protein. However, our analysis of 178 elderly patients, although of reduced sample size, did not reveal an association between CMV antibody levels and mortality.

Our observation, however, does concur with other studies. In a prospective study of 989 patients with documented coronary artery disease and followed for a median of three years, after adjustment for confounders, CMV seropositivity was not related to cardiac mortality. ${ }^{13}$ In a case-control study of 213 elderly patients with a known myocardial infarction or coronary artery disease, CMV seropositivity was also not associated with mortality during a 2-year average follow-up period. ${ }^{12}$ In this study, however, antibodies to herpes simplex type 1 and high titer antibodies to Chlamydia pneumoniae were independent risk factors for death.

It appears that studies that enrolled elderly subjects ( $>70$ years) were those that observed an association between very high antibody titers to CMV and premature mortality., ${ }^{3,5}$ Ours and another study enrolled younger subjects and did not observe reduced mortality associated with high CMV titers. ${ }^{13}$ The average age of our subjects was 58 years. The high antibody titers to CMV may simply reflect failing cellular immunity and T-cell expansion which advances with age and leads to subclinical inflammation, CMV reactivation, and premature death. Those with the highest titers may have the greatest deficit in their cellular immune systems with persistent CMV antigen stimulation and chronic inflammation as reflected by high levels of CMV antibodies and non-specific immune markers such as IL6 or C reactive protein. ${ }^{19}$

Thus the validity of our observation and those of previous studies need confirmation in larger population based studies. This is important because of the ubiquity of CMV infections among world populations and because CMV infections are potentially preventable via immunization and/or treatable with antiviral agents. Currently available data do not support universal immunization against CMV as a means of increasing longevity, but do not exclude the appropriateness of randomized trials of anti-inflammatory or antiviral therapy against CMV for certain subgroups of people, for example, those who are elderly with chronic inflammation and concurrent infections.

\section{References}

1. McVoy MA, Adler SP. Immunologic evidence for frequent age related cytomegalovirus reactivation in seropositive immunocompetent individuals. J Infect Dis 1989; 160:1-10.

2. Chidrawar S, Khan N, Wei W, et al. Cytomegalovirus-seropositivity has a profound influence on the magnitude of major lymphoid subsets within healthy individuals Clin Exp Immunol 2009;155:423-432.
3. Wang GC, Kao WHL, Murakami P, et al. Cytomegalovirus infection and the risk of mortality and frailty in older women: a prospective observational cohort study. Am J Epidemiol 2010;171:1144-52.

4. Strandberg TE, Pitkala KH, Tilvis RS. Cytomegalovirus antibody level and mortality among community-dwelling older adults with stable cardiovascular disease. JAMA 2009;301:380-2.

5. Roberts ET, Haan MN, Dowd JB, Aiello AE. Cytomegalovirus antibody levels, inflammation, and mortality among elderly Latinos over 9 years of follow-up. Am J Epidemiol 2010;172:363-71.

6. Grattan MT. Accelerated graft atherosclerosis following cardiac transplantation: Clinical perspectives. Clin Cardiol 1991; 14:16-20.

7. Zhou YF, Leon MB, Waclawiw MA, et al. Association between prior cytomegalovirus infection and the risk of restenosis after coronary atherectomy. N Engl J Med 1996;335:624-30.

8. Zhu J, Nieto FJ, Horne BD, et al. Prospective study of pathogen burden and risk of myocardial infarction or death. Circulation 2001;103:45-51.

9. Adam E, Probtsfield JL, Burek J, et al. High levels of cytomegalovirus antibody in patients requiring vascular surgery for atherosclerosis. Lancet 1987:2;291-3.

10. Blum A, Giladi M, Weinberg M, et al. High anti-cytomegalovirus (CMV) IgG antibody titer is associated with coronary artery disease and may predict post-coronary balloon angioplasty restenosis. Am J Cardiol 1998;1:866-8.

11. Adler SP, Hur JK, Wang JB, Vetrovec GW. Prior infection with cytomegalovirus is not a major risk factor for angiographically demonstrated coronary artery atherosclerosis. J Infect Dis 1998;177:209-12.

12. Siscovick DS, Schwartz SM, Corey L, et al. Chlamydia pneumoniae, herpes simplex virus type 1, and cytomegalovirus and incident myocardial infarction and coronary heart disease death in older adults: the cardiovascular health study. Circulation 2000:102;2335-40.

13. Blankenberg S, Rupprecht HJ, Bickel C, et al. Cytomegalovirus infection with interleukin-6 response predicts cardiac mortality in patients with coronary artery disease. Circulation 2001;103;2915-21.

14. Pass RF, Zhang C, Evans A, et al. Vaccine prevention of maternal cytomegalovirus infection. N Engl J Med 2009;360:1191-9

15. Adler SP, McVoy M. Detection of cytomegalovirus antibody by enzyme immunoassay and lack of evidence for an effect resulting from strain heterogeneity. J Clin Microbiol 1986;24:870-2

16. Adler SP, McVoy M, Biro VG, et al. 
Detection of cytomegalovirus antibody with latex agglutination. J Clin Microbol 1985;22:68-70.

17. Wang JB, Adler SP, Hempfling SP, et al. Mucosal antibodies to CMV glycoprotein B occur following both natural infection and immunization with human cytomegalovi- ral vaccines. J Infect Dis 1996;174:397-2.

18. Horne BD, Muhlestein JB, Carlquist JF, et al. Statin therapy interacts with cytomegalovirus seropositivity and high c-reactive protein in reducing mortality among patients with angiographically significant coronary disease. Circulation 2003;107:
258-63.

19. Pawelec G, Akbar A. Beverly P, et al. Immunosenescence and cytomegalovirus: where do we stand after a decade? Immunity \& Aging 2010;7:13-8. 